\title{
Levetiracetam Responsive in Late-Onset Idiopathic Paroxysmal Kinesigenic Dyskinesia
}

\author{
Senem Ertugrul' (1), Ferda Selcuk ${ }^{2}$ (1) \\ 'Department of Neurology, Kyrenia University School of Medicine, Kyrenia, Cyprus \\ 2Department of Neurology, Nalbantoglu State Hospital, Nicosia, Cyprus
}

ORCID iDs of the authors: S.E. 0000-000I-9984-74IX; F.S. 0000-0002-2I70-406I.

Cite this article as: Ertugrul S, Selcuk F. Levetiracetam Responsive in Late-Onset Idiopathic Paroxysmal Kinesigenic Dyskinesia. Cyprus J Med Sci 202I; 6(I): 105-6.

\section{CASE PRESENTATION}

A 70-year-old male patient presented to the neurology department of Burhan Nalbantoglu State Hospital, Nicosia, Cyprus, in April 2014 with subtle abnormal movements of the upper and lower limbs. His dyskinesias were triggered by a sudden movement only while getting in or out of the car. During the initial months, attacks consisted of abrupt choreoathetoid movements of the right arm alone; however, subsequent attacks included choreoathetoid movements of the right leg as well. There was no alteration in the level of consciousness. The events lasted 5 to 10 seconds. General physical and neurological examination of the patient produced unremarkable results. Magnetic resonance imaging of the brain, ictal electroencephalographic study, and laboratory studies (complete blood count, urine screen, comprehensive metabolic panel, thyroid function tests, and antithyroid antibodies, antinuclear antibodies, copper, and ceruloplasmin level tests) were negative or within the normal limits. Because the patient was diagnosed with paroxysmal kinesigenic dyskinesia (PKD), mutation of the PRRT2 gene was investigated and was found negative. The patient was treated with $200 \mathrm{mg}$ carbamazepine twice a day, but he was not episode free. Carbamazepine was stopped owing to hyponatremia, and the patient was switched to $500 \mathrm{mg}$ levetiracetam twice a day. The patient achieved complete resolution of signs with levetiracetam monotherapy. On follow-up, the patient was dyskinesia free, and no side effects were reported thereafter.

\section{DISCUSSION}

Paroxysmal dyskinesia (PD) is a rare neurological syndrome characterized by involuntary, intermittent movements manifested by dystonia, chorea, athetosis, ballism, or any combination of these hyperkinetic disorders (I). Demirkiran et al. (2) adopted the term PD and divided it into 4 categories: PKD, paroxysmal nonkinesigenic dyskinesia, paroxysmal exertion-induced dyskinesia, and paroxysmal hypnogenic dyskinesia. Each type has been subdivided into short-lasting ( $\leq 5$ minutes) and long-lasting (>5 minutes) subtypes, and each subtype has been subdivided into idiopathic and secondary (2). Because PKD is clinically relatively uncommon and because the cases reported in the literature mostly occurred at younger ages (I), we therefore reported a late-onset idiopathic PKD in a male patient aged 70 years and a complete resolution of the symptoms with levetiracetam monotherapy.

The etiopathogenesis of PKD is still unclear. The most common type is familial PKD, which is caused by mutation of the PRRT2 gene (3). In light of abnormal electroencephalography results of some patients and the good response to anticonvulsants, PKD is considered a form of reflex epilepsy. The diagnosis of PKD is based on the clinical history. Carbamazepine and phenytoin are usually considered the first-choice drugs $(4,5)$. If they are contraindicated or not effective, other anticonvulsants can be administered (5). The right choice of medications decreases the frequency of attacks or even leads to complete resolution, as in our case. The case we described is an exceptional one because the patient had the first symptoms when he was aged 68 years. The only trigger of his attacks was getting in or out of the car. The duration of his attacks was only up to 10 seconds, and he never lost consciousness. None of his relatives had a similar attack, making our patient the only PKD case in his family. 
Peer-review: Externally peer-reviewed.

Conflict of Interest: Authors have no conflicts of interest to declare.

Financial Disclosure: The authors declared that this study has received no financial support.

\section{REFERENCES}

I. Wei Sun, Jianing Li, Yulan Zhu, Xiaobo Yan, Weizhi Wang. Clinical features of paroxysmal kinesigenic dyskinesia: Report of 24 cases. Epilepsy Behav 2012; 25(4): 695-9. [Crossref]
2. Demirkiran M, Jankovic J. Paroxysmal dyskinesias: clinical features and classification. Ann Neurol 1995; 38(4): 57I-9. [Crossref]

3. Chen WJ, Lin Y, Xiong ZQ, Wei W, Ni W, Tan GH, et al. Exome sequencing identifies truncating mutations in PRRT2 that cause paroxysmal kinesigenic dyskinesia. Nat Genet 20II; 43(I2): 1252-5. [Crossref]

4. Bruno MK, Hallett M, Gwinn-Hardy K, Sorensen B, Considine E, Tucker $\mathrm{S}$, et al. Clinical evaluation of idiopathic paroxysmal kinesigenic dyskinesia: New diagnostic criteria. Neurology 2004; 63(12): 2280-7. [Crossref]

5. Chillag KL, Deroos ST. Oxcarbazepine use in paroxysmal kinesigenic dyskinesia: report on four patients. Pediatr Neurol 2009; 40(4): 295-7. [Crossref] 